\title{
Extract of Stellera Chamaejasme L. Inhibits the Progression of Hepatocellular Carcinoma by Regulating miR-134-5p and JAK1/STAT3 Pathway
}

\author{
Xiaoqing Huang, ${ }_{1}^{1}$ Yan Chen, ${ }^{2}$ Xia Zhang, ${ }^{3}$ Feimeng Li, ${ }^{1}$ and Haoting $\mathrm{Ye}^{3}$
}

\begin{abstract}
Background: Hepatocellular carcinoma (HCC) poses a growing threat to humans due to poor prognosis. Extract of stellera chamaejasme L. (ESC) is reported to inhibit metastasis of HCC. However, the underlying mechanism of ESC in regulating the progression of HCC needs to be further investigated.

Methods: 3-(4, 5-Dimethylthiazol-2-yl)-2,5-diphenyltetrazolium bromide (MTT) assay was used to measure cell proliferation. Flow cytometry was employed to check cell apoptosis. Transwell assay was conducted to assess the abilities of cell migration and invasion. The protein levels of proliferating cell nuclear antigen, cleaved caspase 3 (c-caspase 3), E-cadherin, janus kinase 1 (JAK1), signal transducer and activator of transcription 3 (STAT3), and phosphorylated STAT3 were detected by Western blot. The interaction between $m i R-134-5 p$ and JAK1 was predicted by starBase, which was verified by the dual-luciferase reporter assay and RNA pull-down assay. The messenger RNA levels of miR-134-5p and JAK1 were determined by quantitative real-time polymerase chain reaction.

Results: The results showed that the higher concentration or the longer time treatment of ESC led to the lower survival rate of HCC cells. Besides, ESC induced apoptosis and impeded migration and invasion of HCC cells. Moreover, downregulation of $m i R-134-5 p$ inverted the effects of ESC-mediated repression on HCC progression. Further studies indicated that miR-134-5p targeted the $3^{\prime}$-untranslated region ( $3^{\prime} \mathrm{UTR}$ ) of JAK1 and reversed JAK1-mediated impacts on HCC progression. Simultaneously, ESC inactivated JAK1/STAT3 pathway by regulating the expression of $m i R-134-5 p$.
\end{abstract}

Conclusion: ESC suppressed HCC progression by upregulating the expression of $m i R-134-5 p$ and blocking JAK1/STAT3 pathway.

Keywords: ESC, HCC, JAK1/STAT3 pathway, miR-134-5p

\section{Introduction}

$\mathbf{H}$ epatocellular carcinoma (HCC) is a typical primary liver malignancy, accounting for about $80 \%$ liver cancer worldwide, ${ }^{1}$ and it is estimated to be the fourth most common cause of cancer mortality. ${ }^{2}$ The morbidity and mortality of liver cancer in China exceeds that of the rest of the world. ${ }^{3}$ Hence, it is urgent to look for new therapeutic approaches and molecular targets for HCC treatment.

Stellera chamaejasme $\mathrm{L}$. is a perennial herb, universally used in traditional Chinese medicine. Asada $\mathrm{Y}$ et al. reported that the extract of stellera chamaejasme L. (ESC) func- tioned in anti-HIV. ${ }^{4} \mathrm{Kim} \mathrm{M}$ et al. found that ESC induced cutaneous wound healing and anti-inflammatory activities. ${ }^{5}$ Recent studies showed that ESC could impede the progression of various human cancers. Liu et al. confirmed that ESC induced lung cancer cell apoptosis. ${ }^{6} \mathrm{Kan}$ et al. reported that ESC inhibited the proliferation of HCC cells. ${ }^{7}$ Another article showed that ESC repressed metastasis of HCC cells. ${ }^{8}$ However, the mechanism of ESC in modulating HCC progression is still unclear and needs to be further studied.

MicroRNAs (miRNAs) are short noncoding RNAs (about 22 nucleotides) and regulate gene expression by targeting the $3^{\prime}$-untranslated region ( $3^{\prime}$ UTR) of messenger RNA (mRNA) at

\footnotetext{
${ }^{1}$ Traditional Chinese Medicine, ${ }^{2}$ State Institution of Drug Clinical Trials, and ${ }^{3}$ Department of Pharmacy, Guangdong Second Provincial General Hospital, Guangzhou, Guangdong, China.

Address correspondence to: Xia Zhang; Department of Pharmacy, Guangdong Second Provincial General Hospital; No. 466, Xinggang Middle Road Haizhu District, Guangzhou, Guangzhou 510317, Guangdong, China E-mail: dingjun273229@163.com
} 
the post-transcriptional level. ${ }^{9}$ Emerging reports have deeply improved the authors' comprehension of the importance of miRNAs in human cancers. ${ }^{10,11}$ MiR-134-5p was reported to be associated with gastric cancer, ${ }^{12}$ cervical cancer, ${ }^{13}$ and nasopharyngeal carcinoma. ${ }^{14}$ Yin et al. demonstrated that $m i R-134-5 p$ exerted a dramatically suppressive effect on HCC progression. ${ }^{15}$ A new study indicated that $m i R-134-5 p$ was upregulated under ESC treatment in HCC cells, ${ }^{8}$ yet the underlying mechanism had not been completely addressed.

Janus kinase 1 (JAK1), a human tyrosine kinase protein, plays a key role in cancer therapy. ${ }^{16,17}$ JAK1 could phosphorylate signal transducer and activator of transcription 3 (p-STAT3) to respond to cytokines and growth factors. The JAK-STAT signaling pathway was reported in lots of cancers, ${ }^{18,19}$ including HCC. ${ }^{20,21}$ Therefore, JAK1 may be an attractive cancer drug target and novel regulators that modulate JAK1 expression require to be defined.

In this research, the authors analyzed the impacts of ESC on proliferation, apoptosis, migration, and invasion of HCC cells. Besides, the potential mechanism of ESC in inhibiting HCC progression was further explored by subsequent experiments.

\section{Materials and Methods}

\section{Preparation of ESC}

The experiment was conducted according to a previous report. ${ }^{8}$ In brief, the stellera chamaejasme L. was extracted using ethanol and the condensed liquid was washed on a polyamide column with $60 \%$ ethanol. After the liquid was dried under vacuum, this final compound was defined as ESC.

\section{Cell culture}

Human HCC cell lines (Hep3B and Huh-7) were purchased from the cell bank of the Chinese Academy of Sciences (Shanghai, China). Dulbecco's modified Eagle's medium (Sigma, St. Louis, MO), containing $5 \% \mathrm{CO}_{2}$ and $10 \%$ fetal bovine serum (FBS) (Sigma), was utilized to culture cells.

\section{Proliferation experiment}

Cell proliferation was evaluated by 3-(4, 5-Dimethylthiazol2-yl)-2,5-diphenyltetrazolium bromide (MTT) assay. Cells were treated with various concentrations of ESC for $48 \mathrm{~h}$ or $20 \mu \mathrm{g} / \mathrm{mL}$ ESC for different time. Twenty microliters of MTT solution $(5 \mathrm{mg} / \mathrm{mL})$ (Sigma) was added to the well to incubate for $4 \mathrm{~h}$. Afterward, $200 \mu \mathrm{L}$ dimethyl sulfoxide (Sigma) was added to the well after discarding medium. Optical density values were examined at $490 \mathrm{~nm}$ wavelength under the microplate reader (Bio-Rad, Richmond, CA).

\section{Flow cytometry assay}

Annexin Apoptosis Detection Kit (Sigma) was hired to assess cell apoptosis following the provided protocols. Briefly, cells were resuspended in binding buffer and then $5 \mu \mathrm{L} \mathrm{An-}$ nexin V-fluorescein isothiocyanate (Annexin V-FITC) and $5 \mu \mathrm{L}$ propidium iodide were added to the buffer for incubating for $10 \mathrm{~min}$ in the dark. The stained cells were analyzed by flow cytometry (Thermo Fisher Scientific, Rockford, IL).

\section{Transwell assay}

Transwell chamber precoated with Matrigel (Corning Life Sciences, Corning, NY) or not was introduced to assess the abilities of cell invasion or migration, respectively. Transfected cells were added to the upper chamber, while the medium containing FBS was added to the lower chamber. The migrated or invaded cells were analyzed at an inverted microscope (MultiskanEX; Lab Systems, Helsinki, Finland) after being incubated for $24 \mathrm{~h}$ and stained with crystal violet.

\section{Western blot analysis}

Proteins from samples were isolated using radio immunoprecipitation assay (RIPA) buffer (Vazyme, Nanjing, China). Pierce ${ }^{\mathrm{TM}}$ Rapid Gold BCA Protein Assay Kit (Thermo Fisher Scientific) was used to check the concentration. Proteins were segregated by $10 \%$ sodium dodecyl sulfate-polyacrylamide gel electrophoresis (SDS-PAGE) and then transferred onto polyvinylidene difluoride (PVDF) membranes (Merck Millipore, Billerica, MA). The membranes were blocked with 5\% skimmed milk for $3 \mathrm{~h}$. After being washed with phosphate buffered saline, the membranes were incubated with the primary antibodies against proliferating cell nuclear antigen (PCNA) (1:3000, ab18197; Abcam, Cambridge, UK), c-caspase 3 (1:1500, ab2302; Abcam), E-cadherin (1:3000, ab40772; Abcam), MMP-2 (1:2000, ab97779; Abcam), MMP-9 (1:1000, ab38898; Abcam), JAK1 (1:2000, ab47435; Abcam), STAT3 (1:3000, ab31370; Abcam), p-STAT3 (1:3000, ab76315; Abcam), or glyceraldehyde 3phosphate dehydrogenase (GAPDH) (1:2500, ab9485; Abcam) overnight. The secondary antibody Goat AntiRabbit IgG H\&L (HRP) (1:3000, ab205718; Abcam) was added for incubating for $3 \mathrm{~h}$ after being rewashed again. The protein bands were visualized using the ChemiDoc ${ }^{\mathrm{TM}}$ MP Imaging System (Bio-Rad) after being treated with ECL kit (Vazyme).

\section{RNA isolation and quantitative real-time polymerase chain reaction}

RNAiso Plus kit (Takara, Dalian, China) was introduced to extract RNA from cells according to the provided steps and then RNA was reversed to cDNA by PrimeScript ${ }^{\mathrm{TM}} \mathrm{RT}$ Master Mix kit (Takara). The quantitative real-time polymerase chain reaction (qRT-PCR) was conducted by Fast SYBR $^{\mathrm{TM}}$ Green Master Mix kit (Vazyme). The data were analyzed by using $2^{-\Delta \Delta C t}$ method. Beta-actin ( $\beta$-actin) and U6 were introduced as the endogenous controls. Primers in this study were as follows:

miR-134-5p (forward 5'-ACAATGCGCTGACCTAGCT GTA-3', reverse 5'-CAGTCCACCAAGCAGTCACAG-3'); JAK1 (forward 5'-CGCTCTGGGAAATCTGCT-3', reverse, $5^{\prime}$-TGATGGCTCGGAAGAAAGG-3'); $\beta$-actin (forward 5'-GCACCACACCTTCTACAATG-3', reverse, 5'-TGCTT GCTGATCCACATCTG-3'); and U6 (forward, 5'-TCCGGG TGATGCTTTTCCTAG-3', reverse, 5'-CGCTTCACGAATT TGCGTGTCAT-3').

\section{Cell transfection}

The miR-134-5p mimic (miR-134-5p) and miR-134-5p nhibitor (anti-miR-134-5p), as well as corresponding controls 
(miR-NC and anti-miR-NC), were obtained from GenePharma (Shanghai, China). JAK1 expression plasmid (JAK1) and its control (Vector) were acquired from RiboBio (Guangzhou, China). Transfection experiments were performed by utilizing Lipofectamine 2000 reagent (Invitrogen, Carlsbad, CA) following the given procedures.

\section{Dual-luciferase reporter assay}

The potential target sites of $m i R-134-5 p$ and JAK1 were predicted by starBase. ${ }^{22}$ The sequences of wild-type JAK1 (JAK1 3'UTR-WT) and its mutant (JAK1 3'UTR-MUT) harboring putative target sites of $m i R-134-5 p$ were cloned and inserted into pGL3 vectors (Promega, Madison, WI). Then the vectors with $m i R-134-5 p$ or miR-NC were cotransfected into Huh-7 and Hep3B cells using Lipofectamine 2000 (Invitrogen). The luciferase activity was measured by the DualGlo ${ }^{\circledR}$ Luciferase Assay System (Promega).

\section{RNA pull-down assay}

Biotin-labeled probe against miR-134-5p (Bio-miR-134$5 \mathrm{p})$ and its negative control (Bio-NC) were obtained from Sangon (Shanghai, China). After being incubated with cell lysates, the probes were captured by streptavidin-coupled beads (Sangon). Proteinase $\mathrm{K}$ was used to digest proteins and the enrichment of JAK1 was checked by qRT-PCR.

\section{Statistical analysis}

Experimental data were presented by mean \pm standard deviation. Two independent groups were compared by Student's $t$-test. For more than two groups, the one-way analysis of variance (ANOVA) followed by Turkey's test was used to assess the difference. Each experiment was conducted at least three times independently. $p<0.05$ represented statistical significance.

\section{Results}

ESC inhibited proliferation, migration, and invasion, and induced apoptosis of HCC cells

To explore the function of ESC in HCC progression, the authors first checked the cytotoxicity of ESC on HCC cells. The results showed that the cell survival rate gradually decreased with the increasing ESC concentrations and a notable downtrend was observed at $20 \mu \mathrm{g} / \mathrm{mL}$ ESC (Fig. 1A, B). These data indicated that $20 \mu \mathrm{g} / \mathrm{mL}$ ESC exerted inhibition effect on cell viability and the cell survival rate was $\sim 80 \%$, and $20 \mu \mathrm{g} / \mathrm{mL}$ ESC exhibited a low cytotoxicity in HCC cells. Thus, they chose $20 \mu \mathrm{g} / \mathrm{mL}$ ESC for the subsequent experiments. They then investigated the effect of ESC on HCC cells under different treatment time, and the result indicated that cell survival rate was significantly declined under the treatment of 24, 48, and $72 \mathrm{~h}$ compared with control (Fig. 1C, $p<0.05)$. Apoptosis assay showed that ESC conspicuously promoted apoptosis of HCC cells (Fig. 1D, $p<0.05$ ). In addition, transwell assay elucidated that ESC repressed migration and invasion of HCC cells (Fig. 1E, F, $p<0.05$ ). Next, the protein levels of PCNA (related to proliferation), c-caspase 3 (related to apoptosis), and E-cadherin (related to metastasis) were measured and the data illustrated that ESC markedly downregulated the expression of PCNA and upregulated the levels of c-caspase 3 and E-cadherin (Fig. 1G-I, $p<0.05$ ). These data revealed that $20 \mu \mathrm{g} / \mathrm{mL}$ ESC significantly inhibited the expression of MMP-2 and MMP-9 in both Hep3B and Huh-7 cells, suggesting that ESC inhibited cells migration and invasion by downregulating MMP-2 and MMP-9 expression (Supplementary Fig. S1A, B, $p<0.05$ ). Together, these results suggested that ESC could impede the progression of HCC cells in vitro.

\section{ESC elevated the expression of miR-134-5p in HCC cells}

A previous report indicated that $m i R-134-5 p$ was highly expressed under ESC treatment. ${ }^{8}$ To investigate the relationship between ESC and miR-134-5p, the authors checked the expression of $m i R-134-5 p$ in HCC cells treated with ESC. The results showed that the level of $m i R-134-5 p$ continued to increase with increasing ESC concentrations (Fig. 2A, B, $p<0.05$ ). Similarly, miR-134-5p was upregulated under treatment of ESC with different time $(24,48$, and $72 \mathrm{~h}$ ) compared with control (Fig. 2C, D, $p<0.05$ ). All in all, these results manifested that ESC enhanced the expression of $m i R-134-5 p$ in HCC cells in vitro.

\section{ESC repressed $H C C$ progression by regulating the expression of $\mathrm{miR}-134-5 \mathrm{p}$}

To further study the relationship of the two in HCC, the authors evaluated the level of $m i R-134-5 p$ in HCC cells treated with ESC, anti-miR-134-5p, or ESC+anti-miR-134$5 \mathrm{p}$, as well as the corresponding controls. The data showed that $m i R-134-5 p$ was remarkedly upregulated in ESC group, whereas the expression was significantly reduced in anti-miR-134-5p and ESC+anti-miR-134-5p group (Fig. 3A, $p<0.05)$. MTT assay indicated that downregulation of $m i R-134-5 p$ transposed the effect of ESC-mediated inhibition on proliferation of HCC cells (Fig. 3B, C, $p<0.05$ ). Besides, the effect of ESC-mediated promotion on apoptosis was reversed by downregulating miR-134-5p (Fig. 3D, $p<0.05$ ). Furthermore, $m i R-134-5 p$ inhibitor rescued the inhibitory effect of ESC on migration and invasion of HCC cells (Fig. 3E, F, $p<0.05$ ). Meanwhile, the expression levels of three proteins (PCNA, c-caspase 3 , and E-cadherin) in the ESC group were reversed following the transfection with anti-miR-134-5p (Fig. 3G-J, $p<0.05)$. From these results, it could be concluded that ESC suppressed proliferation, migration, and invasion, and induced apoptosis of HCC cells by modulating the expression of $m i R-134-5 p$ in vitro.

\section{MiR-134-5p targeted the 3UTR of JAK1 and negatively regulated the expression of JAK1}

To figure out the potential mechanism of $m i R-134-5 p$ in $\mathrm{HCC}$, starBase was hired to find the possible target genes of $m i R-134-5 p$. The result showed that $m i R-134-5 p$ could bind to the $3^{\prime}$ UTR of JAK1 (Fig. 4A). To verify the prediction, corresponding reporter plasmids (JAK1 3'UTRWT and JAK1 3'UTR-MUT) were constructed and data showed that $m i R-134-5 p$ apparently diminished the luciferase activity of JAK1 3'UTR-WT in HCC cells, rather than JAK1 3'UTR-MUT (Fig. 4B, C, $p<0.05$ ). 

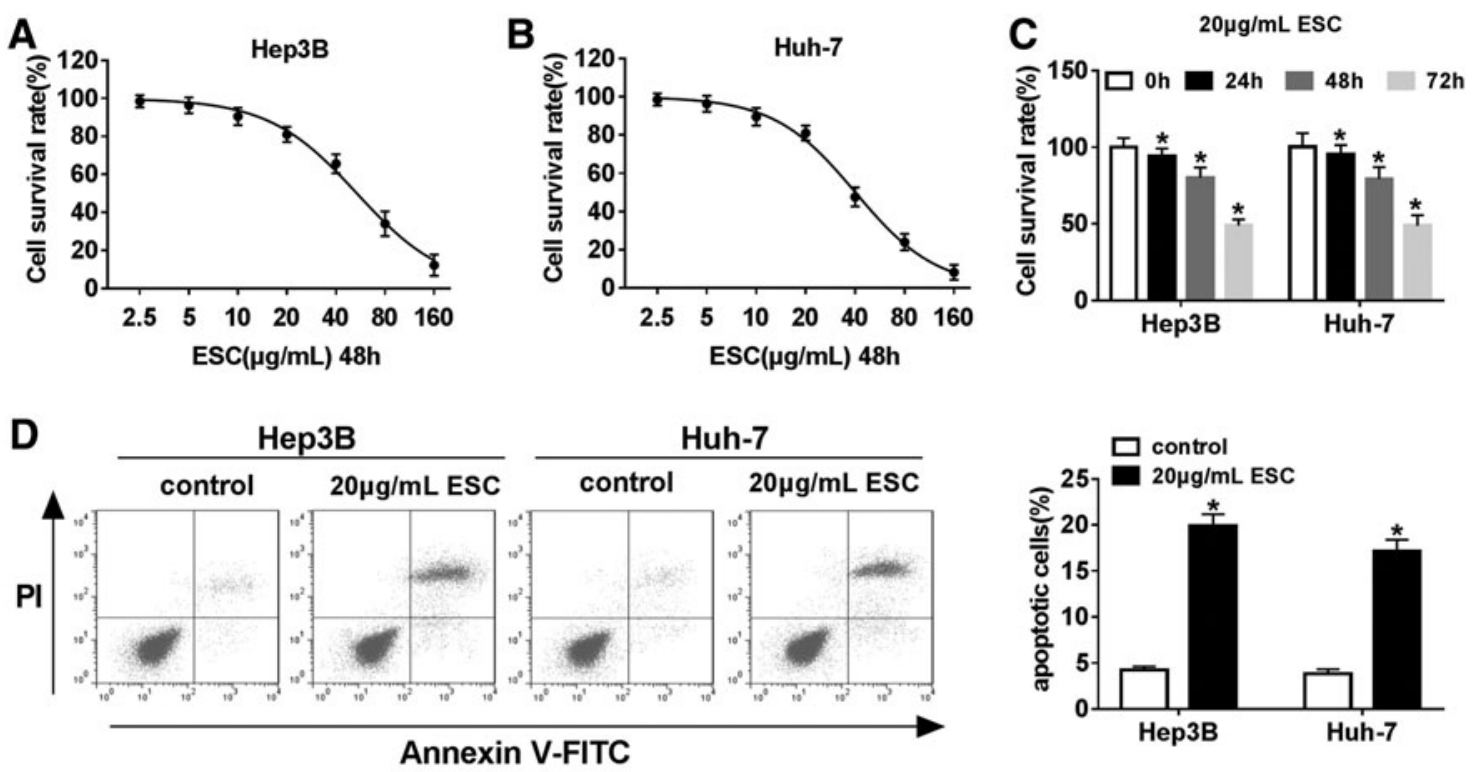

$\mathbf{E}$

Huh-7
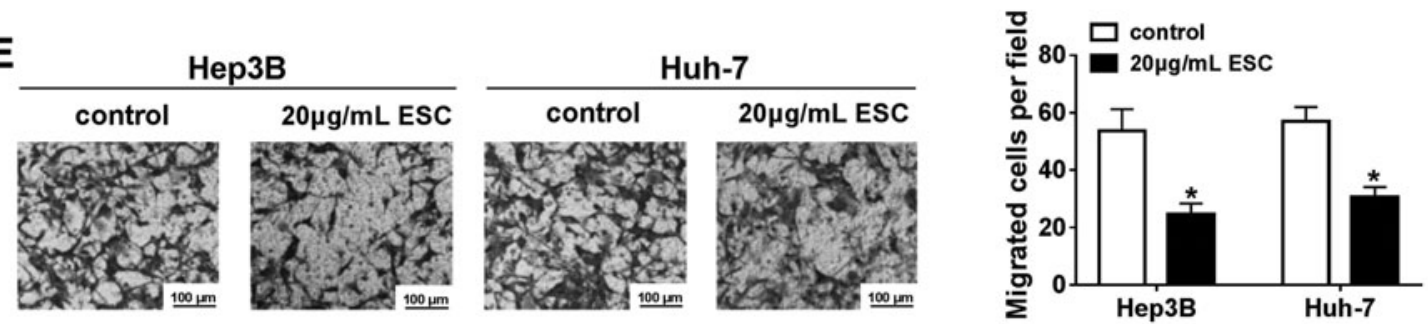

$\mathbf{F}$

Huh-7
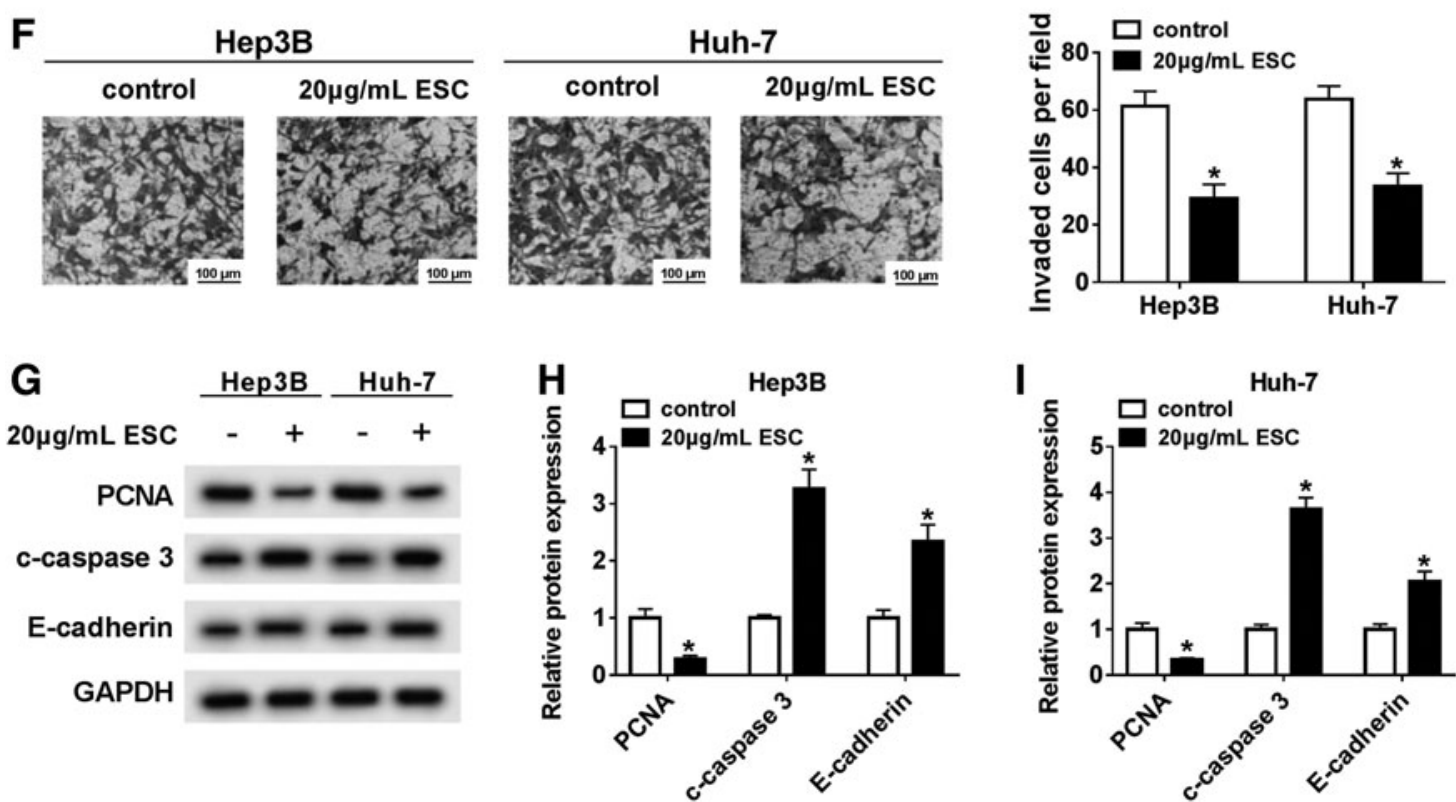

FIG. 1. ESC repressed proliferation, migration, and invasion, and promoted apoptosis. (A-C) The proliferation of HCC cells under ESC treatment was assessed by MTT assay. (D) The apoptosis of HCC cells treated with ESC was checked by flow cytometry. (E, F) Transwell assay was employed to measure the abilities of cell migration and invasion. (G-I) The protein levels of PCNA, c-caspase 3, and E-cadherin in HCC cells treated with ESC were evaluated by Western blot. ${ }^{*} p<0.05$. ESC, extract of stellera chamaejasme L.; HCC, hepatocellular carcinoma; MTT, 3-(4, 5-Dimethylthiazol-2-yl)-2, 5-Diphenyltetrazolium Bromide; PCNA, proliferating cell nuclear antigen. 
A

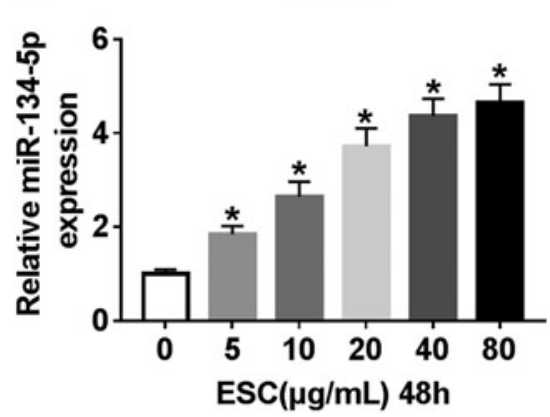

FIG. 2. MiR-134-5p was upregulated under ESC treatment. (A, B) The expression of $m i R-134-5 p$ in HCC cells treated with different ESC concentrations was detected by qRT-PCR. (C, D) The expression of $m i R-134-5 p$ in $\mathrm{HCC}$ cells treated under ESC with different time was detected by qRT-PCR. ${ }^{*} p<0.05$. qRT-PCR, quantitative real-time polymerase chain reaction.

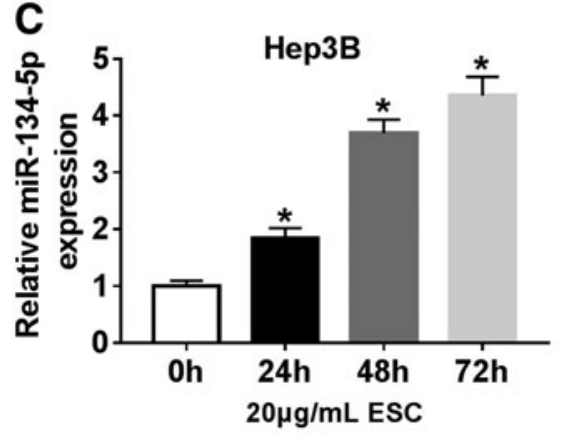

Huh-7
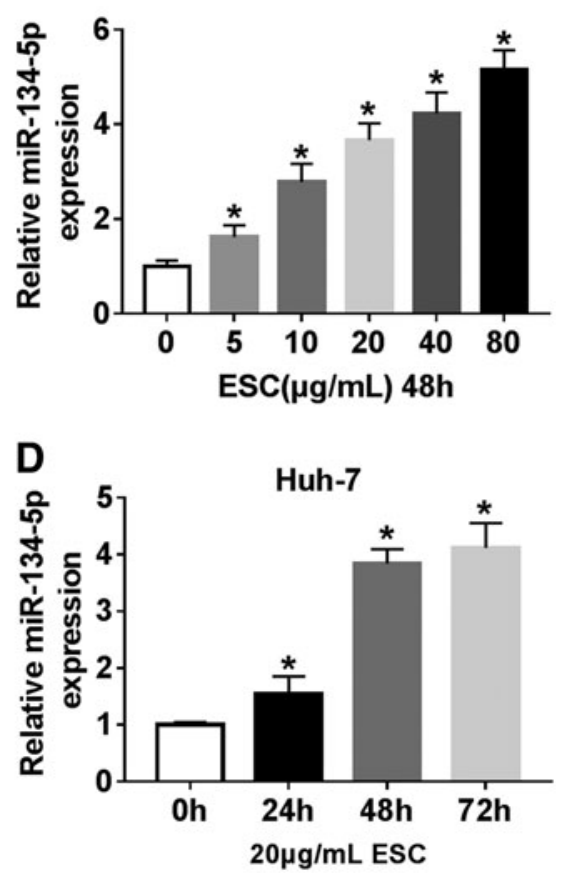

Simultaneously, RNA pull-down assay confirmed that $m i R$ 134-5p was able to precipitate JAK1 in Hep3B and Huh-7 cells (Fig. 4D, E, $p<0.05$ ). Further research indicated that upregulation of $m i R-134-5 p$ significantly reduced the mRNA and protein levels of JAK1 in HCC cells, whereas downregulation of $m i R-134-5 p$ obviously increased the expression of JAK1 (Fig. 4F-I, $p<0.05$ ). Taken together, these results illustrated that $m i R-134-5 p$ could target the $3^{\prime} \mathrm{UTR}$ of JAK1 and negatively modulated the mRNA and protein levels of JAK1 in vitro.

\section{Upregulation of $\mathrm{miR}-134-5 \mathrm{p}$ reversed the impact of JAK1-mediated promotion on HCC progression}

To understand the role of the interaction between $m i R$ 134-5p and JAK1 in HCC progression, the authors checked the expression of JAK1 in HCC cells transfected with JAK1 or JAK1+miR-134-5p, as well as the matched controls. The data showed that JAK1 was evidently upregulated in JAK1 group, while the expression level of JAK1 was clearly overturned after the transfection with miR-134-5p (Fig. 5A$\mathrm{C}, p<0.05)$. In addition, elevated expression of $m i R-134-5 p$ rescued the effect of JAK1-mediated promotion on proliferation of HCC cells (Fig. 5D, E, $p<0.05$ ). Apoptosis assay elucidated that $m i R-134-5 p$ mimic revoked the inhibitory effect of JAK1 on apoptosis of HCC cells (Fig. 5F, $p<0.05$ ). Moreover, upregulating the expression of $m i R-134-5 p$ abolished the effects of JAK1-mediated enhancement on migration and invasion of HCC cells (Fig. 5G, H, $p<0.05$ ). Afterward, the protein levels of PCNA, c-caspase 3, and E-cadherin were checked in transfected HCC cells and the results disclosed that the expression of these proteins in JAK1 group was inverted after the transfection with $m i R$ 134-5p (Fig. 5I-L, $p<0.05$ ). To sum up, these results demonstrated that $m i R-134-5 p$ inhibited cell proliferation, migration, invasion and promoted cell apoptosis by regulating JAK1 in HCC cells.

\section{ESC repressed $H C C$ progression by regulating the expression of JAK1}

To further investigate the role of JAK1 signaling in ESCtreated HCC cells, Hep3B and Huh-7 cells were treated with ESC and transfected with JAK1 or Vector. As shown in Supplementary Figure S2A and B, ESC significantly inhibited the expression of JAK1, which was restored by JAK1 overexpression $(p<0.05)$. MTT assay suggested that JAK1 overexpression reversed the inhibitory effect of ESC on cell proliferation in both Hep3B and Huh-7 cells (Supplementary Fig. S2D, E, $p<0.05)$. Moreover, the promotion effect of ESC on cell apoptosis was blocked by upregulating JAK1 (Supplementary Fig. S2F, $p<0.05$ ). ESC significantly suppressed cell migration and invasion, which were hindered by JAK1 upregulation (Supplementary Fig. 2G, H, $p<0.05)$. Besides, the inhibition on PCNA expression and promotion on c-capase-3 and E-cadherin expression of ESC were reversed by overexpression of JAK1 in both Hep3B and Huh-7 cells (Supplementary Fig. S2I, J, $p<0.05$ ). These findings indicated that ESC inhibited HCC progression by downregulating JAK1 expression.

\section{ESC modulated JAK1/STAT3 pathway through regulating $\mathrm{miR}-134-5 \mathrm{p}$}

JAK1 is a core member of the JAK1-STAT3 pathway. To explore whether ESC could affect JAK1/STAT3 pathway, the relative expression of JAK1 and p-STAT3/STAT3 in treated HCC cells was measured. The results showed that the expression levels of JAK1 and p-STAT3/STAT3 conspicuously declined under the treatment with ESC, whereas 
A

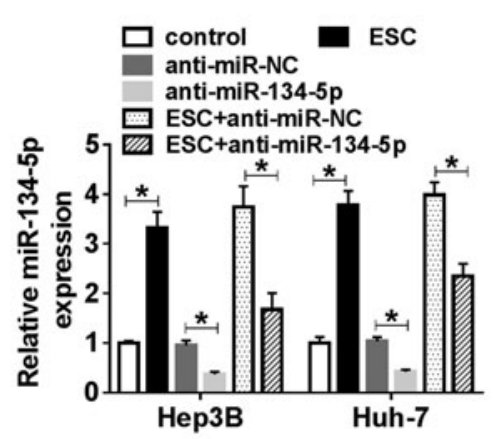

D

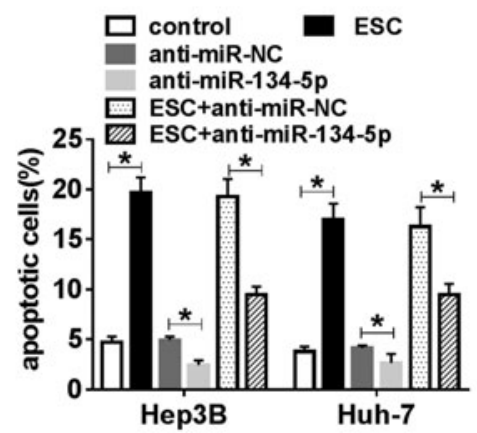

G

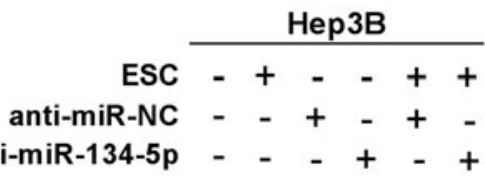

PCNA

c-caspase 3

E-cadherin

GAPDH
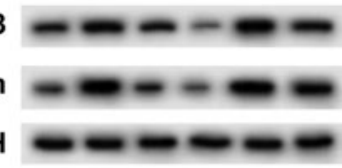

J

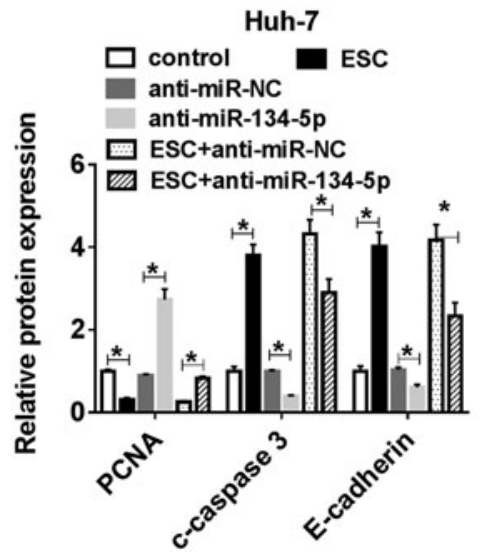

B

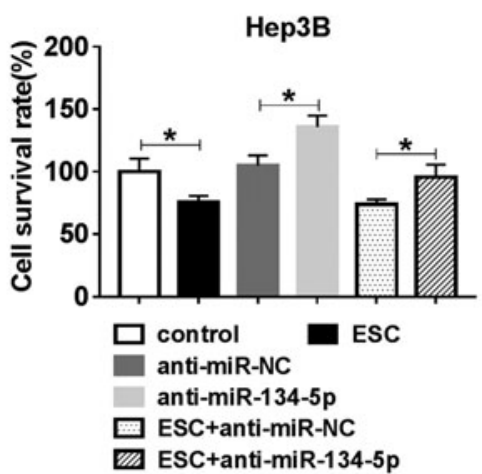

E

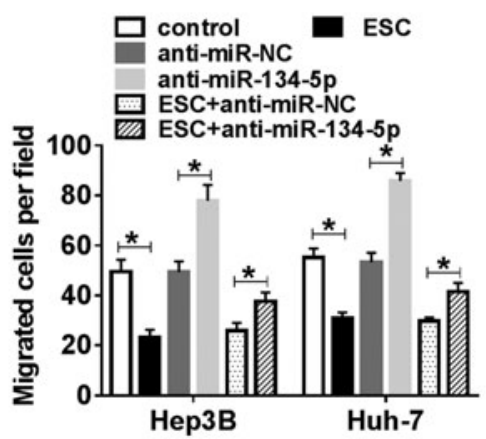

H $\square \underset{\text { anti-miR-NC }}{\text { control }} \stackrel{\text { Hep3B }}{\text { ESC }}$

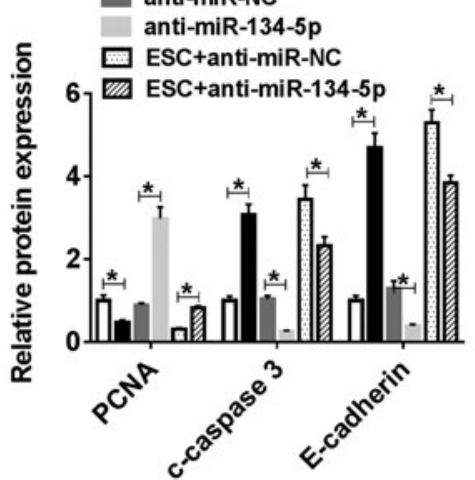

C

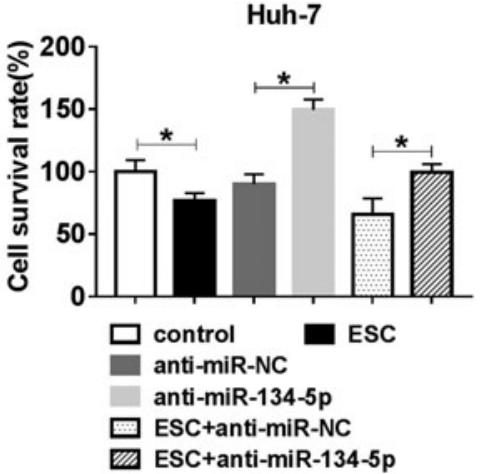

F

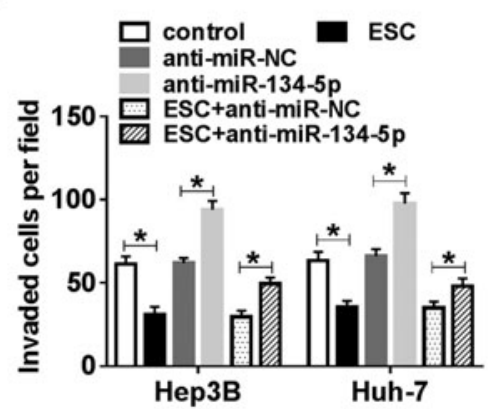

I

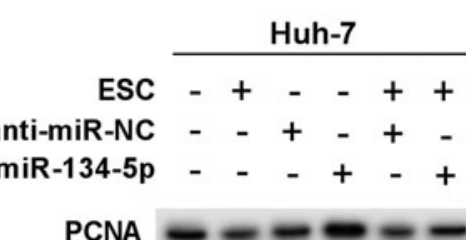

c-caspase 3

E-cadherin

GAPDH

FIG. 3. MiR-134-5p inhibitor reversed ESC-mediated effects on HCC progression. (A) The level of miR-134-5p in HCC cells treated with ESC, anti-miR-134-5p, or ESC+anti-miR-134-5p, as well as their matched controls, was measured by qRTPCR. (B, C) The proliferation of HCC cells treated with ESC was checked by MTT assay. (D) Flow cytometry was introduced to assess apoptosis of treated HCC cells. (E, F) The abilities of migration and invasion of treated HCC cells were evaluated by transwell assay. (G-J) The expression levels of PCNA, c-caspase 3, and E-cadherin in treated HCC cells with ESC were determined by Western blot. $* p<0.05$. 
A

Target site: chr1:65300127-65300132

$\begin{array}{ll}\text { JAK1 3'UTR-WT } & \text { 5' GUGUUCUGUCCAAAAAGUCACU 3' } \\ \text { miR-134-5p } & \text { 3' GGGGAGACCAGUUGGUCAGUGU 5' }\end{array}$

JAK1 3'UTR-MUT 5' GUGAGGCAUCAGGAAUUGAGAU 3'
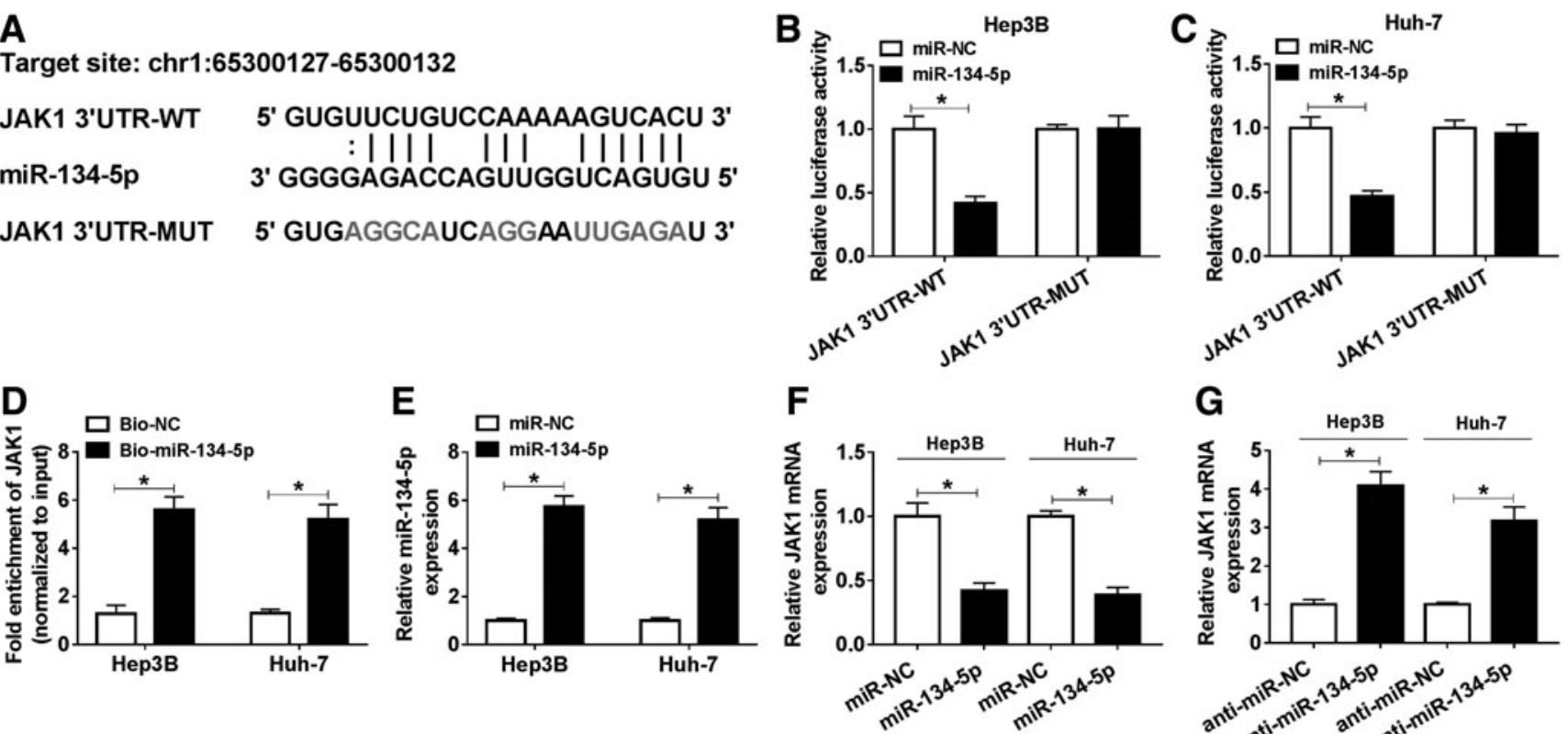

H

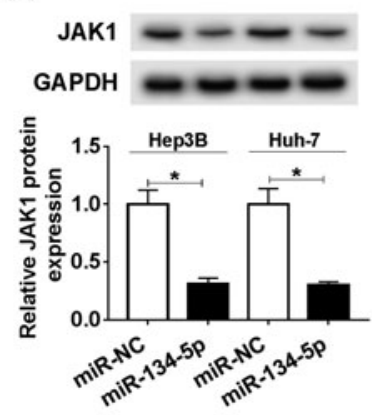

I

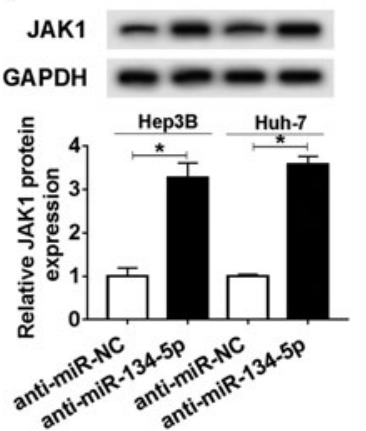

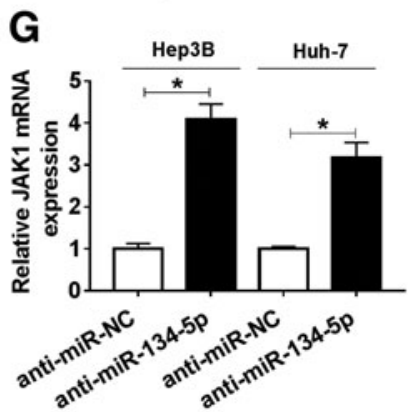

FIG. 4. $m i R-134-5 p$ targeted the $3^{\prime} \mathrm{UTR}$ of JAK1 in HCC cells. (A) The interaction between $m i R-134-5 p$ and JAK1 was forecasted by starBase. (B, C) The dual-luciferase reporter assay was used to check the luciferase activity of HCC cells cotransfected with miR-134-5p or miR-NC and JAK1 3'UTR-WT or JAK1 3'UTR-MUT. (D, E) RNA pull-down assay was performed and the relative enrichment of JAK1 in samples was detected by qRT-PCR. (F, G) The mRNA level of JAK1 in HCC cells transfected with $m i R-134-5 p$ or anti-miR-134-5p, as well as corresponding controls, was measured by qRT-PCR. $(\mathbf{H}, \mathbf{I})$ The protein level of JAK1 in transfected HCC cells was checked by Western blot. * $p<0.05$. 3'UTR, 3'-untranslated region; mRNA, messenger RNA; JAK1, janus kinase 1.

the effect was reversed by downregulating miR-134-5p (Fig. 6A, B, $p<0.05$ ). Collectively, these results explicated a fact that ESC could inactivate JAK1/STAT3 pathway by upregulating $\mathrm{miR}$-134-5p.

\section{Discussion}

HCC is a growing threat to humans and is responsible for cancer-associated deaths worldwide. ${ }^{23}$ Therefore, it is essential to find more anti-HCC drugs and investigate the molecular mechanisms behind them, which will contribute to the development of more effective treatments for HCC.

ESC is widely utilized in traditional Chinese medicine and confirmed to possess antibacterial, ${ }^{24}$ antivirus, ${ }^{25}$ and antitumors activities. ${ }^{6,7}$ To explore the effects of ESC on HCC progression, the authors first detected its cytotoxicity to HCC cells and found that ESC inhibited cell proliferation in a dose- and time-dependent manner, and cell sur- vival rate had a sharp downtrend at $20 \mu \mathrm{g} / \mathrm{mL}$ ESC. They then chose this concentration for the next experiments. Apoptosis assay and transwell assay indicated that ESC significantly promoted apoptosis and repressed migration and invasion of HCC cells, respectively. Meanwhile, ESC notably decreased the protein level of PCNA and increased the levels of c-caspase 3 and E-cadherin. These results suggested that ESC indeed hindered the development of HCC cells in vitro.

Abnormal expression of miRNA was often observed in many cancers. ${ }^{26,27}$ Recently, an article reported that the expression of many miRNAs was changed under ESC treatment in $\mathrm{HCC}^{8}$ Hence, the level of $m i R-134-5 p$ in $\mathrm{HCC}$ cells treated with ESC was determined and the data illustrated that the expression of $m i R-134-5 p$ was positively correlated with ESC concentration or treatment time. That $m i R-134-5 p$ was upregulated under ESC treatment was in line with Liu's report. ${ }^{8}$ Next, the function of $m i R-134-5 p$ in HCC progression was studied and the results manifested 
A
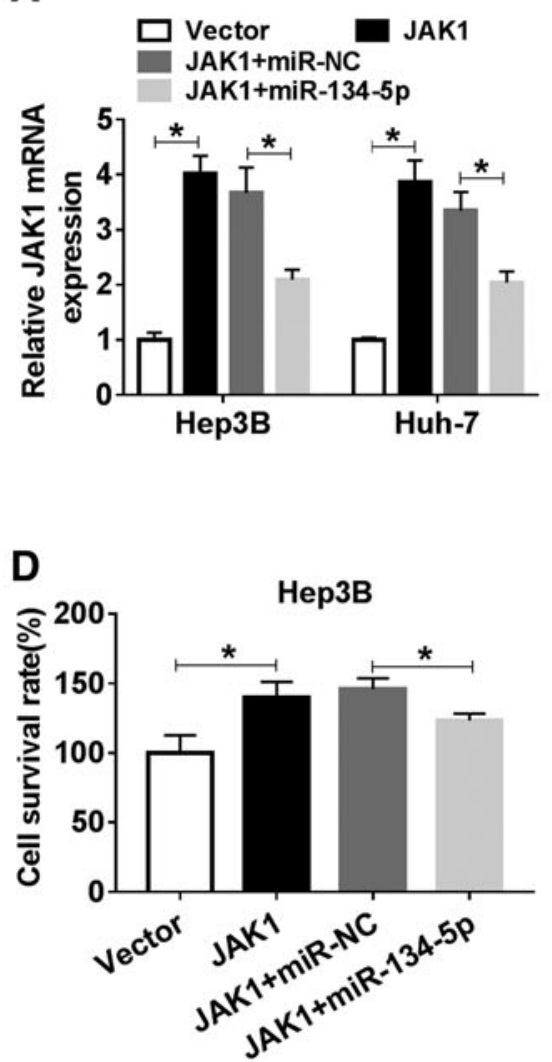

G

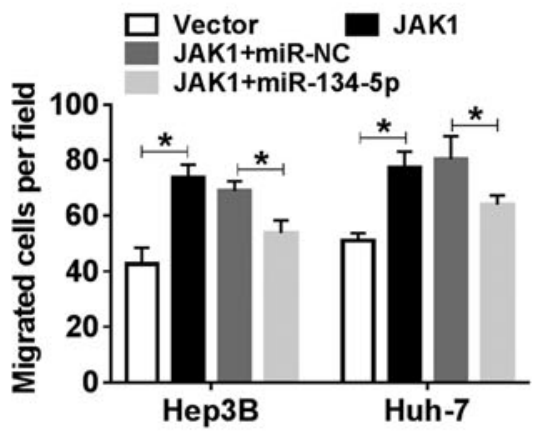

J

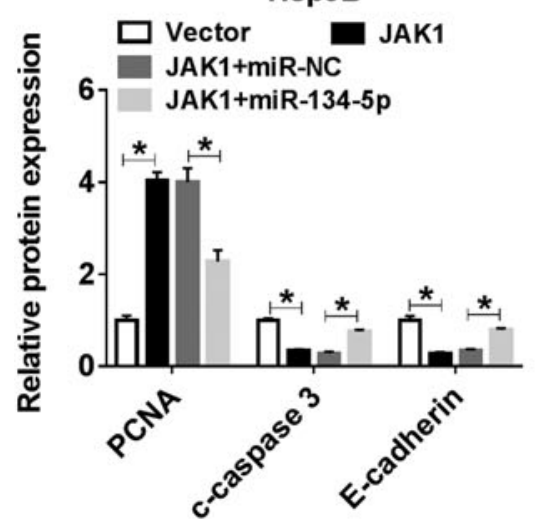

B
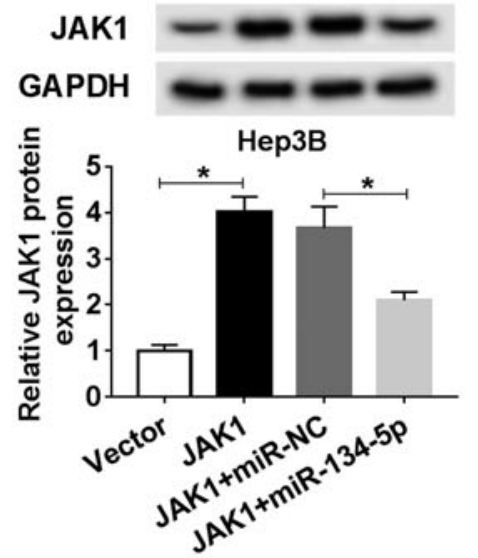

E

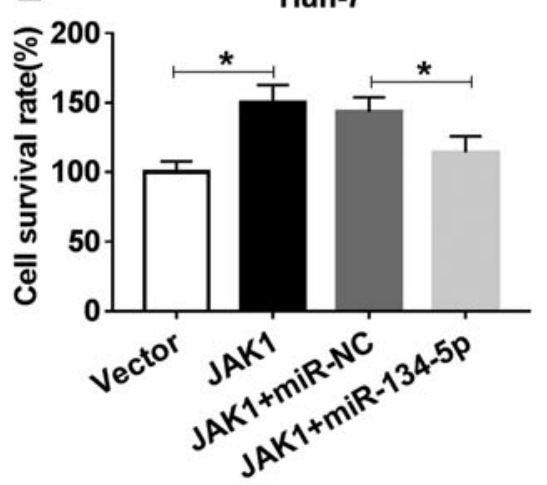

H

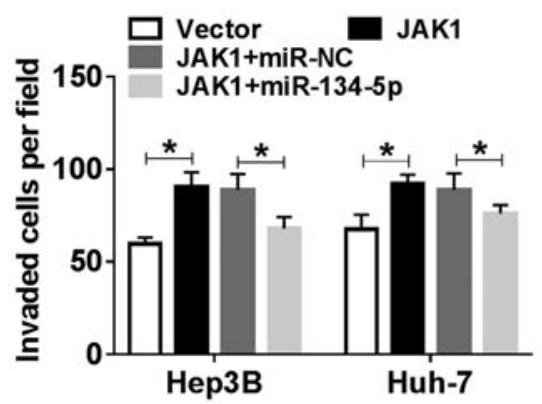

K

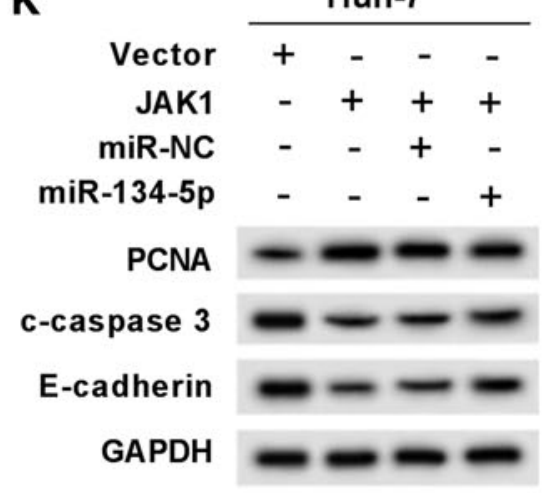

C

JAK1

GAPDH

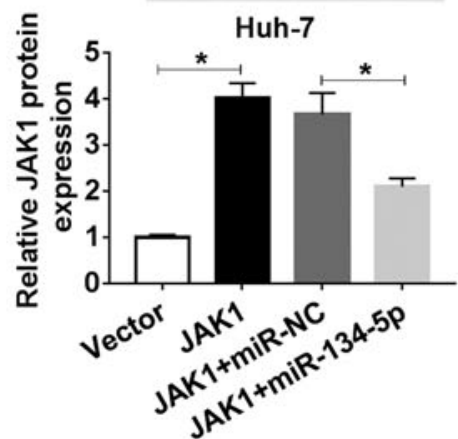

F
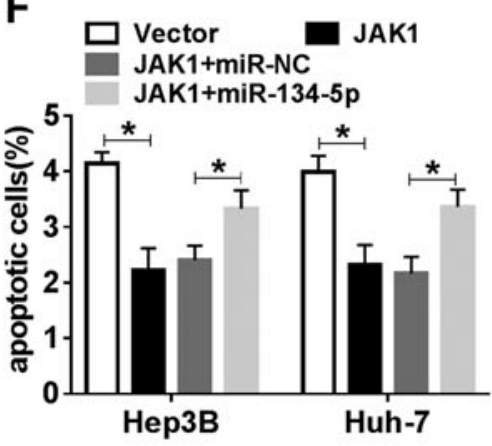

I

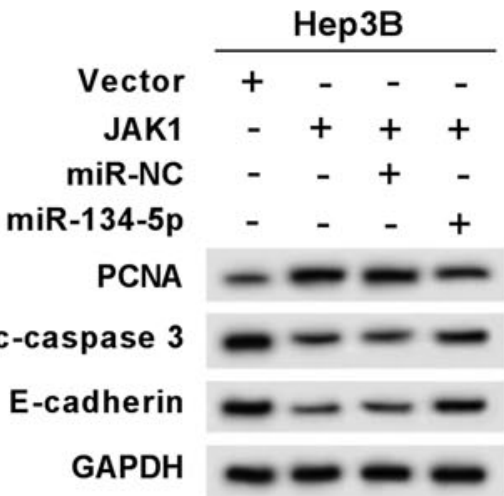

L

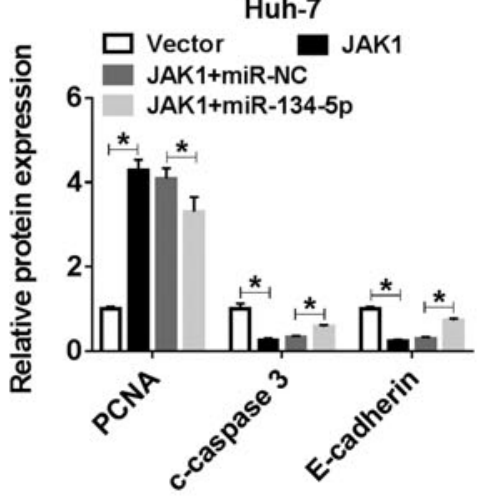

FIG. 5. Upregulation of miR-134-5p inverted JAK1-mediated effects on proliferation, apoptosis, migration, and invasion of HCC cells. (A-C) The mRNA and protein levels of JAK1 in HCC cells transfected with JAK1 or JAK1+miR-134-5p, as well as matched controls, were assessed by qRT-PCR and Western blot, respectively. (D, E) The proliferation of transfected HCC cells was checked by MTT assay. (F) Flow cytometry was used to measure cell apoptosis. (G, H) Transwell assay was utilized to evaluate the abilities of migration and invasion. (I-L) The expression of PCNA, c-caspase 3, and E-cadherin in transfected HCC cells was checked by Western blot. $* p<0.05$. 

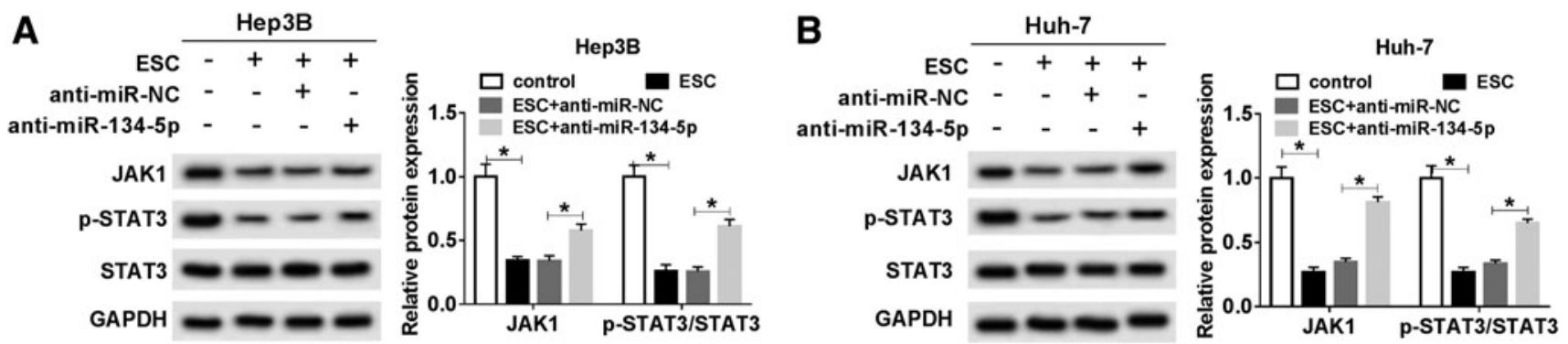

FIG. 6. ESC reduced the expression of proteins associated with JAK1/STAT3 pathway by miR-134-5p. (A) The protein levels of JAK1, p-STAT3, and STAT3 in Hep3B cells treated with ESC or ESC+anti-miR-134-5p, as well as corresponding controls, were detected by Western blot. (B) The protein levels of JAK1, p-STAT3, and STAT3 in treated Huh-7 cells were measured by Western blot. ${ }^{*} p<0.05$. p-STAT3, phosphorylated signal transducer and activator of transcription 3 .

that downregulation of $m i R-134-5 p$ promoted proliferation, migration, and invasion, and inhibited apoptosis of HCC cells. Similarly, the protein levels of PCNA, c-caspase 3, and E-cadherin in HCC cells treated with ESC were also reversed after the transfection with anti-miR134-5p. Together, these results elucidated that ESC damaged HCC progression by upregulating miR-134-5p in vitro.

To further explore the underlying mechanism of $m i R$ 134-5p in HCC progression, bioinformatics analysis was performed to find its possible target genes. The data showed JAK1 was a target of miR-134-5p and the interaction was proved by dual-luciferase reporter assay and RNA pull-down assay. Also, miR-134-5p negatively modulated the expression of JAK1 in HCC cells. In addition, overexpression of JAK1 boosted proliferation, migration, and invasion, and hampered apoptosis of HCC cells, whereas elevating the expression of $m i R-134-5 p$ inverted these impacts. Growing evidence has emphasized the fact that JAK1/STAT3 pathway plays a pivotal role in human cancers. Chen et al. found that HOXA10 deteriorated gastric cancer through activating JAK1/STAT3 pathway. ${ }^{28}$ Zhou et al. reported that CtBPs promoted the malignancy of osteosarcoma cells through JAK1/STAT3 pathway. ${ }^{29}$ From the results above, the authors knew that ESC regulated the expression of $m i R-134-5 p$ and $m i R-134-$ $5 p$ could bind to the $3^{\prime} \mathrm{UTR}$ of JAK1, which inspired the curiosity to investigate whether ESC and $m i R-134-5 p$ were involved in JAK1-STAT3 pathway. The protein levels of JAK1, STAT3, and p-STAT3 in treated HCC cells were measured and the results indicated that ESC strikingly weakened the levels of JAK1 and p-STAT3/STAT3, while downregulation of $m i R-134-5 p$ reversed the effects. In summary, ESC upregulated miR-134-5p that interacted with and downregulated JAK1, thereby reducing the level of p-STAT3/STAT3, ultimately leading to the inactivation of JAK1-STAT3 pathway.

\section{Conclusions}

In conclusion, this research demonstrated that ESC could inhibit the progression of HCC by upregulating miR-134-5p and inactivating miR-134-5p-mediated JAK1-STAT3 pathway. This new mechanism may facilitate the development of novel therapeutic approaches for HCC.

\section{Authors' Contributions}

X.Z. and X.H. conceptualized the study. Y.C. and X.H. collected the data. F.L. and H.Y. analyzed the data. All the authors revised and approved the final version.

\section{Disclosure Statement}

No competing financial interests exist.

\section{Funding Information}

This work was approved by Special fund for medical and health development on inheriting and developing of Traditional Chinese Medicine in 2018 (Guangdong Traditional Chinese Medicine Office [2018] No.5).

\section{Supplementary Material}

Supplementary Figure S1

Supplementary Figure S2

\section{References}

1. El-Serag HB, Rudolph KL. Hepatocellular carcinoma: Epidemiology and molecular carcinogenesis. Gastroenterology 2007;132:2557.

2. Fitzmaurice C, Allen C, Barber RM, et al. Global, regional, and national cancer incidence, mortality, years of life lost, years lived with disability, and disability-adjusted life-years for 32 cancer groups, 1990 to 2015: A systematic analysis for the global burden of disease study. JAMA Oncol 2017; 3:524.

3. Yang JD, Hainaut P, Gores GJ, et al. A global view of hepatocellular carcinoma: Trends, risk, prevention and management. Nat Rev Gastroenterol Hepatol 2019;16:589_ 604.

4. Asada Y, Sukemori A, Watanabe T, et al. Stelleralides A-C, novel potent anti-HIV daphnane-type diterpenoids from Stellera chamaejasme L. Org Lett 2011;13:2904.

5. Kim M, Lee HJ, Randy A, et al. Stellera chamaejasme and its constituents induce cutaneous wound healing and antiinflammatory activities. Sci Rep 2017;7:42490.

6. Liu X, Zhu X. Stellera chamaejasme L. extract induces apoptosis of human lung cancer cells via activation of the death receptor-dependent pathway. Exp Ther Med 2012;4: 605 . 
7. Kan XX, Li Q, Chen X, et al. A novel cell cycle blocker extracted from Stellera chamaejasme L. inhibits the proliferation of hepatocarcinoma cells. Oncol Rep 2016;35:3480.

8. Liu X, Wang S, Xu J, et al. Extract of Stellerachamaejasme L(ESC) inhibits growth and metastasis of human hepatocellular carcinoma via regulating microRNA expression. BMC Complement Altern Med 2018;18:99.

9. Jovanovic M, Hengartner MO. miRNAs and apoptosis: RNAs to die for. Oncogene 2006;25:6176.

10. Ma L, Teruya-Feldstein J, Weinberg RA. Tumour invasion and metastasis initiated by microRNA-10b in breast cancer. Nature 2007;449:682.

11. Mathonnet G, Fabian MR, Svitkin YV, et al. MicroRNA inhibition of translation initiation in vitro by targeting the cap-binding complex eIF4F. Science 2007;317:1764.

12. Lario S, Brunet-Vega A, Quílez ME, et al. Expression profile of circulating microRNAs in the Correa pathway of progression to gastric cancer. United European Gastroenterol J 2018;6:691.

13. Zhang WY, Liu YJ, He Y, et al. Suppression of long noncoding RNA NCK1-AS1 increases chemosensitivity to cisplatin in cervical cancer. J Cell Physiol 2019;234:4302.

14. Liu Y, Tao Z, Qu J, et al. Long non-coding RNA PCAT7 regulates ELF2 signaling through inhibition of miR-134-5p in nasopharyngeal carcinoma. Biochem Biophys Res Commun 2017;491:374.

15. Yin $\mathrm{C}$, Wang $\mathrm{PQ}, \mathrm{Xu} \mathrm{WP}$, et al. Hepatocyte nuclear factor$4 \alpha$ reverses malignancy of hepatocellular carcinoma through regulating miR-134 in the DLK1-DIO3 region. Hepatology 2013;58:1964.

16. Patel SJ, Sanjana NE, Kishton RJ, et al. Identification of essential genes for cancer immunotherapy. Nature 2017; 548:537.

17. Shin DS, Zaretsky JM, Escuin-Ordinas H, et al. Primary resistance to PD-1 blockade mediated by JAK1/2 mutations. Cancer Discov 2017;7:188.

18. Chuang $\mathrm{CH}$, Greenside PG, Rogers ZN, et al. Molecular definition of a metastatic lung cancer state reveals a targetable CD109-Janus kinase-Stat axis. Nat Med 2017;23: 291.
19. Johnson DE, O'Keefe RA, Grandis JR. Targeting the IL-6/JAK/STAT3 signalling axis in cancer. Nat Rev Clin Oncol 2018;15:234.

20. Calvisi DF, Ladu S, Gorden A, et al. Ubiquitous activation of Ras and Jak/Stat pathways in human HCC. Gastroenterology 2006;130:1117.

21. Yoshikawa H, Matsubara K, Qian GS, et al. SOCS-1, a negative regulator of the JAK/STAT pathway, is silenced by methylation in human hepatocellular carcinoma and shows growth-suppression activity. Nat Genet 2001;28:29.

22. Li JH, Liu S, Zhou H, et al. starBase v2.0: Decoding miRNA-ceRNA, miRNA-ncRNA and protein-RNA interaction networks from large-scale CLIP-Seq data. Nucleic Acids Res 2014;42:D92.

23. Bruix J, Qin S, Merle P, et al. Regorafenib for patients with hepatocellular carcinoma who progressed on sorafenib treatment (RESORCE): A randomised, double-blind, placebo-controlled, phase 3 trial. Lancet 2017;389: 56.

24. Jo BG, Park NJ, Jegal J, et al. A new flavonoid from Stellera chamaejasme L., stechamone, alleviated 2,4dinitrochlorobenzene-induced atopic dermatitis-like skin lesions in a murine model. Int Immunopharmacol 2018;59: 113.

25. Yan M, Lu Y, Chen CH, et al. Stelleralides D-J and AntiHIV Daphnane Diterpenes from Stellera chamaejasme. J Nat Products 2015;78:2712.

26. Hua X, Xiao Y, Pan W, et al. miR-186 inhibits cell proliferation of prostate cancer by targeting GOLPH3. Am J Cancer Res 2016;6:1650.

27. Qingping M, Qianqian J, Qiang P, et al. MicroRNA-143 inhibits migration and invasion of human non-small-cell lung cancer and its relative mechanism. Int J Biol Sci 2013; 9:680.

28. Chen W, Wu G, Zhu Y, et al. HOXA10 deteriorates gastric cancer through activating JAK1/STAT3 signaling pathway. Cancer Manag Res 2019;11:6625.

29. Wang P, Yu B, Wang C, et al. C-terminal of E1A binding protein 2 promotes the malignancy of osteosarcoma cells via JAK1/Stat3 signaling. J Cell Commun Signal 2019;14: 67-76. 